\title{
ADVANCED COMPUTER-BASED APPROACHES FOR FOOD QUALITY EVALUATION: UPDATED REVIEW
}

\author{
P. Veleva-Doneva* \\ Department of Informatics and Mathematics, Faculty of Economics Trakia University, Stara Zagora, \\ Bulgaria
}

\begin{abstract}
From the point of view of their usage biological resources must be managed in a sustainable manner in order to uphold the social, economic and environmental functions and to contribute to the peoples' livelihoods. Within the food production and processing industry requirements are focused on improvement of quality control of technologies by incorporating them into precise, computer-based, low-cost methods. The present work is a review of different approaches and tools for computer aided methods for food quality evaluation. Powerful techniques based on Near Infrared Spectroscopy and Aquaphotomics are presented.
\end{abstract}

Key words: Aquaphotomics, NIR Spectroscopy, multivariate data analysis, quality control, food safety

\section{INTRODUCTION}

Lately the good level of public health has become one of the major tasks for improving the quality of human life. The public interest referring to good-quality and production methods of food and food control has significantly enhanced in the last decades. The strategies of many countries are targeted to determine regulatory standards against food fraud, low quality, bacterial control, etc. Their rules are related to hygienic production, storage and transportation. In the meantime, recent researches showed numerous cases of poisoning with ready-to-consumption products dangerous to their users. $[8,9,13,16,29]$. The classical techniques for food control are objective and precise but require enormous effort. They are time-consuming, expensive and destructive. The requirement for building innovative engineering methodology for food quality control used directly in farms, on food production lines, is growing. Quick responses are particularly significant in industry, where a solution of some problems is required within minutes. That might allow for appropriate corrective actions, directed toward protecting people's health to be taken in a timely manner $[4,6,32,40]$.

\footnotetext{
*Correspondence to: Petya Veleva-Doneva, Department of Informatics and Mathematics, Faculty of Economics Trakia University, Stara Zagora, Bulgaria, tel: 042699 439; 042699 438, E-mail:pveleva@uni-sz.bg
}

Near-infrared (NIR) spectroscopy opens an innovative area in food science, biotechnology, and engineering by examining and describing biological systems via a quick non-destructive monitoring of their interaction with NIR light. This technique is based on the absorption of $\mathrm{C}-\mathrm{H}, \mathrm{N}-\mathrm{H}, \mathrm{O}-\mathrm{H}$, and $\mathrm{S}-\mathrm{H}$ molecular bonds that are contained in most raw materials and products and uses the diapason from 780 to $2500 \mathrm{~nm}$ of the electromagnetic spectrum [28, $31]$.

Near-infrared spectroscopy is a flexible technology and has lots of advantages. It allows samples in various physical phases solids, liquids, pasty and gases, to be measured directly with no pre-treatment. Water has less absorbance in the NIR region compared to absorbance in the mid-infrared one. Thus, aqueous solutions, food or biological samples, which generally contain lots of water, might be measured directly without complicated sample preparation procedures and waste materials such as toxic solvents. Samples could be measured directly through the glass cuvettes, glass vials or even through transparent packaging. Remote measurements using quartz fiber optics are possible and could be used for on-line process monitoring. Due to the nondestructive nature of the analysis, the samples can be used for additional tests. An extra advantage of NIR spectroscopy is that spectra often contain information regarding physical 
properties such as temperature, viscosity, density, particle size, etc.

Effective usage of NIR spectroscopy depends on the appropriate selection of spectral instruments. Choosing the instrument may vary according to the products analyzed their chemical composition and structure, analytical accuracy, reliability and implementation requirements. Modular - configurable instruments with a range of light sources, fibers and accessories for various measurement modes allow quick and easy measurement of particular type of products. Fiber optic probes are a very useful solution for in-vivo testing, evaluating large samples, inspecting real-time reactions, and some application where it is hard to bring the sample to the spectrometer. The flexibility and usability make these instruments one of the most broadly used tools in present spectroscopy. Fiber-optic probes have the broadest application in laboratory and on-line examination of different food samples such as raw milk, dairy products, meat, meat products, fruit, beverages, etc. [1, 3, 10, 18, 25, 33, 21, 24, 27]. Progress in optical electronics leads to miniaturization of NIR instruments. The lately proposed cheap, portable with small size NIR spectrometers have lots of advantages for non-destructive, online, or in-situ analysis in agro-food industry [32].

Over the last fifteen years NIR spectroscopy has been used together with an innovative tool called Aquaphotomics. This method has been developed by prof. Roumiana Tsenkova from Kobe University, Japan. It can be used to determine water hydrogen bonds in various aqueous systems under different perturbations. The aim is to utilize water transformations and to obtain more information to understand changes in the observed living systems on a bio-molecular level [37]. Twelve characteristic wavelengths named "WAter MAtrix CoordinateS" (WAMACS) have been observed in the range of the first overtone of the water NIR spectra, where despite the different type of perturbation the examined systems showed predictable spectral variations.

Transformations of water absorbance patterns can be charted as a star called "Aquagram" [38]. Aquaphotomics has been applied in numerous fields as water characterization, food quality control, early diagnostics in medicine, etc. [19, 20, 22, 35]. A lot of publications linked to water quality evaluation can be found [2, 14, 30, 34, 36].
The application of Aquaphotomics based on spectral data for analytical aims depends on multivariate data analysis. Today this is of much more importance because of power increase of computers and improvement of NIR spectrophotometers that allows large number of spectral data to be obtained. Chemometrics is an analytical procedure very often used in Aquaphotomics. This method uses mathematical and statistical models for extracting important information from analytical data [15].

The purpose of this article is to reveal the practicality of NIR spectroscopy and Aquaphotomics for rapid and non-destructive quality control of various food products.

Application of NIR spectroscopy and Aquaphotomics in food quality control

NIR spectroscopy has been successfully applied by Huang et al., [11] for intramuscular fat investigation in fresh, frozen and frozenthawed pork meat. They achieve the best results in the first derivative of the raw spectra and the application of Gabor filtered mean spectra. Wu et al., [41] have applied Partial least squares regression (PLSR) to create a prediction model in pork meat exposed to freeze-thawed processes with correlation coefficient of prediction of 0.81 and root mean square error of 0.33 . A comparable analytical procedure has been applied by Xionq et al., [42] with regression coefficients in prediction (Rp) of 0.944 and root mean squared errors estimated by prediction (RMSEP) of 0.081 . Li et al. [17] have examined meat pork quality to distinguish normal from pale, soft and exudative meat (PSE). Niu at al. [24] have applied NIR spectra to build a classification and prediction models for identification of donkey, beef, pork and mutton meat by SIMCA method. Accuracy of $100 \%$ for calibration and $98 \%$ for prediction has been attained. A spectral instrument has been used by Collell et al. [5] in prediction of water activity and moisture content in fermented dry sausages.

The author's objective in this paper was to present reliable models for rapid and nondestructive quality detection of Bulgarian white brined cheese and Bulgarian yellow cheese from cow milk in different stage of ripening and models for detecting the origin of different types of honey by applying NIR spectral analysis in combination with chemometrics and aquaphotomics. 
MATERIAL AND METHODS

\section{Sample preparation}

Three series of experiments were conducted. In the 1st experiment (Exp-1) white brine cheese were made from cow milk using a classical white brine cheese making scheme, according to Bulgarian national standard. Samples for analysis were taken after 3, 5, 7, 9 and 12 days of ripening.

In the 2nd experiment (Exp-2) Bulgarian yellow cheese were made from cow milk using a classical semi-hard cheese making scheme, according to Bulgarian national standard for Bulgarian yellow cheese. Samples for analysis were taken after $1,15,20,25,30,35,40$ and 45 days of ripening.

In the 3rd experiment (Exp-3) representative samples of unifloral (sunflower, acacia, linden and coriander), multifloral and honeydew honey were investigated. The origin of the honey samples was determined by pollen analysis.

\section{NIR spectra acquisition and data processing} Diffuse reflection spectra of all tested food samples were obtained with a portable scanning NIRQuest 512 instrument in the range 900-1700 $\mathrm{nm}$ using petri dish. The multivariate spectral analysis was carried out by Pirouette ver. 4.5 (Infometrix, Inc., Woodinville, WA, USA) software program. Prior to analysis the spectra were transformed using: averaging of 3 consecutive spectra, autoscaling for each wavelength and multiple scatter correction (MSC).

Descriptive univariate statistical calculations were performed using the Box-and-Whisker plot [39] method to analyze the recorded physical parameters of the food samples. Savitzky-Golay smoothing [23] with $2^{\text {nd }}$ order polynomials and 11 points was used as spectral pretreatment which followed by multiplicative scatter correction (MSC) transformation [23] to remove the possible baseline differences. Principal component analysis (PCA) [7] was used to describe multidimensional patterns of the NIR dataset and to discover outliers.

The absorbance values at specific water matrix coordinates (WAMACs) [37] define the water spectral pattern (WASP) in food samples. The visualization of WASP was done by a radar chart $[12 ; 38]$. The aquagram displays the MSC transformed, normalized and averaged absorbance values of different sample groups at selected wavelengths [38]. Aquagrams were calculated at selected wavelengths of the 2 nd overtone of $\mathrm{OH}$, which showed highest importance in the loadings of PCA which showed peaks in difference spectra. The values of water matrix coordinates for aquagram Aq $\lambda$ were calculated using the following equation:

$A q_{\lambda}=\frac{A_{\lambda}-\mu_{\lambda}}{\sigma_{\lambda}}$,

where $A_{\lambda}$ is absorbance after multiplicative scatter correction (MSC), $\mu_{\lambda}$ is the mean value of all spectra, and $\sigma_{\lambda}$ is standard deviation of all spectra at wavelength $\lambda$, respectively.

Water matrix coordinates at $1344,1364,1372$, $1382,1398,1410,1439,1445,1464,1474$, 1493 and $1518 \mathrm{~nm}$, were used for axes. R programing language [26] was used for calculation and visualization.

\section{RESULTS AND DISCUSSION}

The aquagram, calculated using spectral data of tested samples, are presented at Figures 1-3. The WAMACS coordinates cover the most distinctive species of water structure. For all investigated samples, significant differences in aquagrams, connected with quality of the food samples, were observed.

Differences in absorption spectra and respective aquagrams for (Exp-1 and Exp-2) could be explained with different functionally structures of water in different stage of ripening for Bulgarian brine cheese and Bulgarian yellow cheese. As can be seen from the aquagrams (Figure 1 and Figure 2) at first ripening days in both cases $\left(3^{\text {-rd }}\right.$ and $5^{\text {th }}$ days for the Exp-1 and $1^{\text {-st }}, 15^{\text {th }}$ and $20^{\text {th }}$ days for the Exp-2) the samples contain approximately $75 \%-80 \%$ of water. In both cases changes in water content and proportion of free and bounded water occurred. Changes in water content in Bulgarian brine cheese (Exp-1) can be seen at $7^{\text {-rd }}, 9^{\text {th }}$ and $12^{\text {th }}$ days of ripening (Figure 1). For Bulgarian yellow cheese (Exp2) the sagnificant changes in water content can be seen at $40^{\text {th }}$ and $45^{\text {th }}$ days of ripening (Figure 2).

As can be seen at Figure 3 the aquagrams for Bulgarian honey from different origin are very dissimilar (Exp-3). The coriander and linden honey aquagrams are similar but they are different from acacia honey aquagram. The multifloral, sunflower and honeydew honey aquagrams are very similar but they are completely different from the others. All these aquagrams can be used to make classification models for recognition different types of honey based on their origin. 


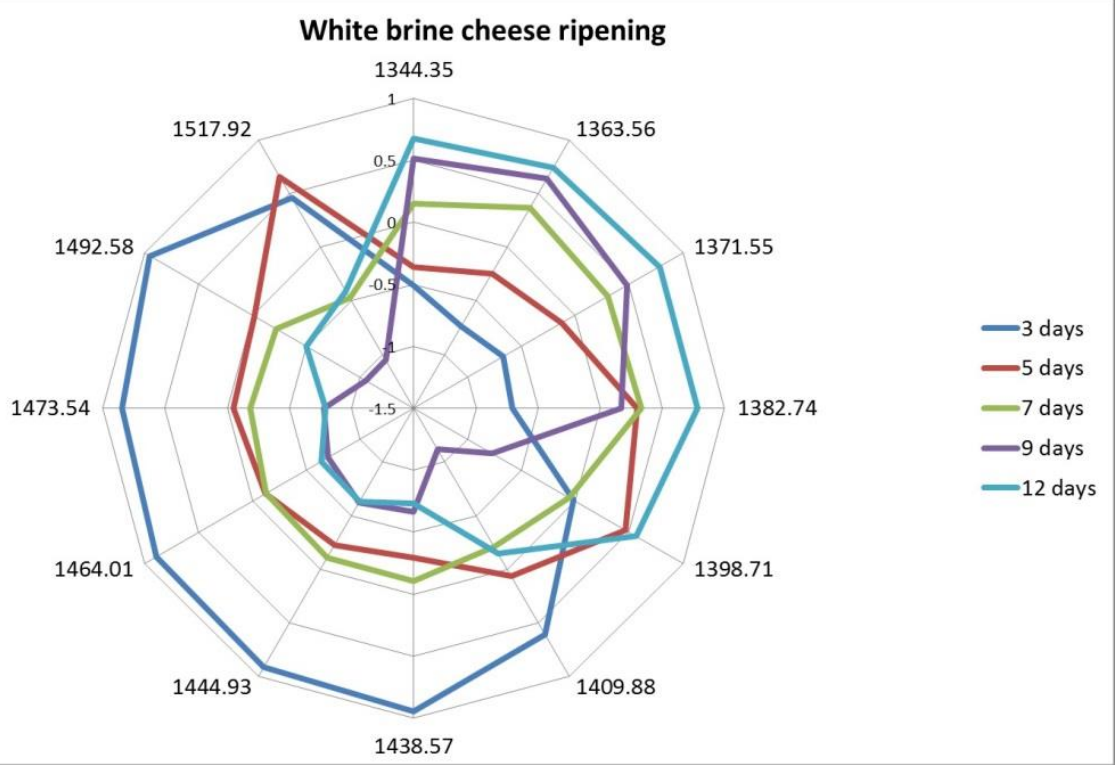

Figure 1. Aquagram for Bulgarian white brined cheese (from cow milk) ripening

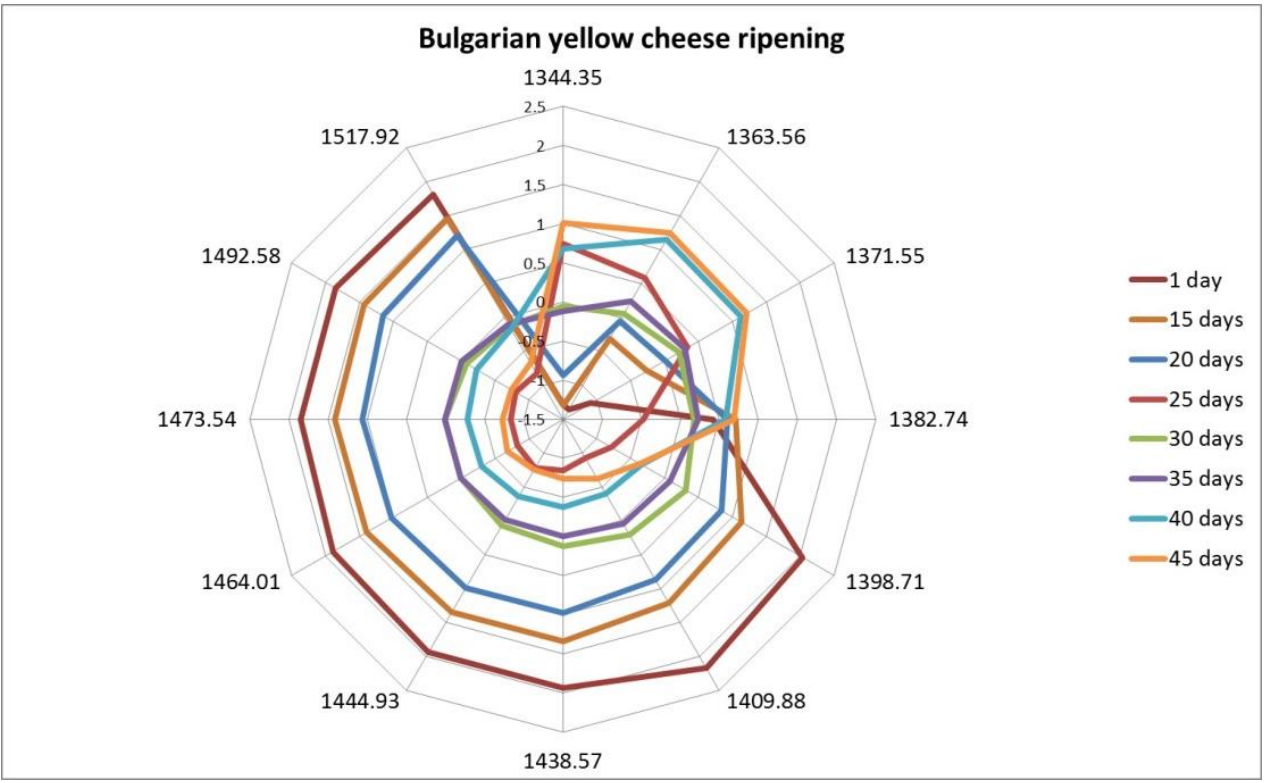

Figure 2. Aquagram for Bulgarian yellow cheese (from cow milk) ripening

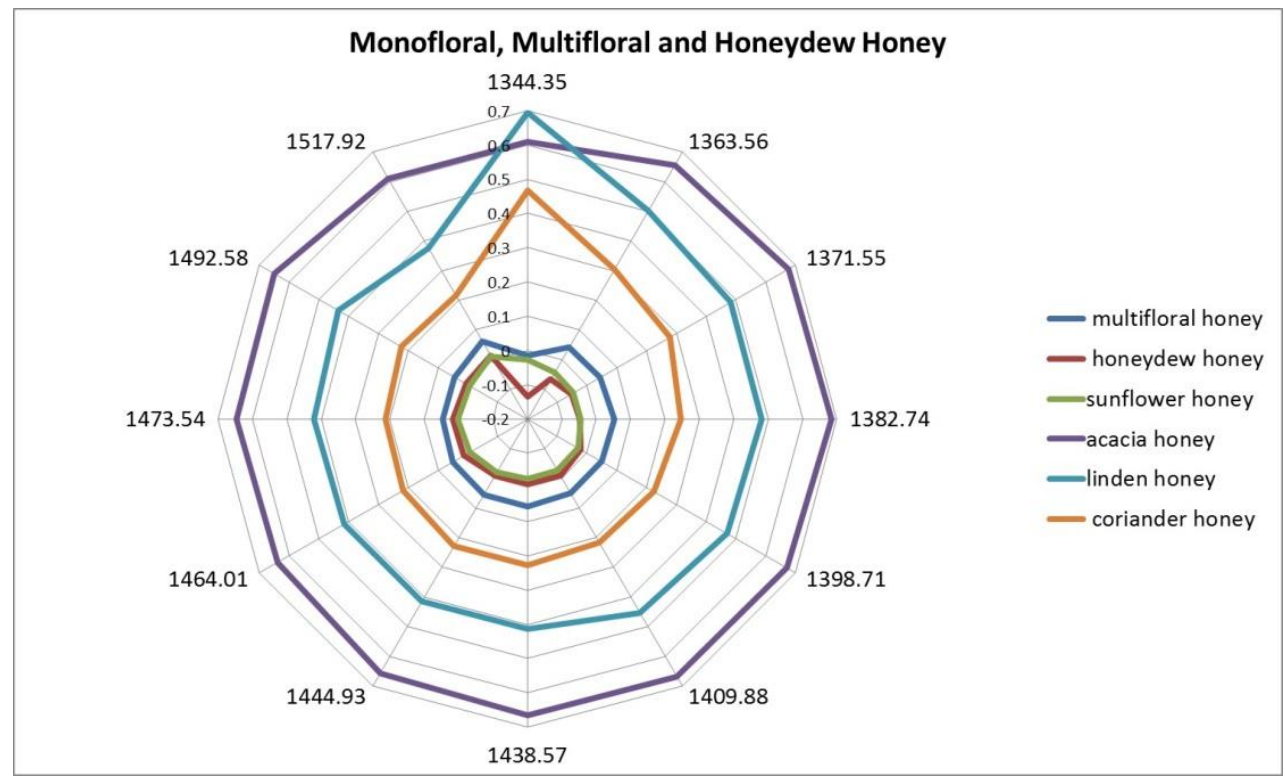

Figure 3. Aquagram for Bulgarian honey from different origin 


\section{CONCLUSIONS}

In the present study, it has been demonstrated that NIR technique coupled with aquaphotomics concept is useful method for monitoring of food quality through observation of water absorbance bands contained into the food products. It has been demonstrated that the absorption regions of water provided the most important spectral information about Bulgarian brine cheese and Bulgarian yellow cheese quality based on the stages of ripenig. Aquagrams for all tested types of honey showed clear differences connected to their origin.

NIR technique and aquaphotomics based on the molecular water mirror concept may provide a quick and accurate alternative for classical analytical measurements and can be used for qualytative modeling for food control.

\section{REFERENCES}

1. Balage, J.M., da Luz, E. Silva, S., Gomide, C.A., Bonin, M.de N., Figueira, AC, Predicting pork quality using Vis/NIR spectroscopy. Meat Sci., 108: 37-43, 2015.

2. Bázár, G., Kovács, Z., Tanaka, M. and Tsenkova, R., Aquaphotomics and its extended water mirror concept explain why NIRS can measure low concentration aqueous solutions, In: Aquaphotomics, "Understanding Water in Biological World", The 5th Kobe University Brussels European Centre Symposium "Innovation, Environment, and Globalisation", Brussels, Belgium.

http://www.aquaphotomics.com/pdfposters/nr2.pdf, 2014.

3. Berzaghi, P. and Riovanto, R., Near infrared spectroscopy in animal science production: principles and applications. Ital. J .Anim. Sci., 8 (3): 39-62, 2009.

4. Cheng, J.H., Dai, Q., Sun, D.W., Zeng, X.A., Liu, D. and Pu, H.B., Applications of non-destructive spectroscopic techniques for fish quality and safety evaluation and inspection. Trends in Food Science \& Technology, 34:18-31, 2013.

5. Collell, C., Gou, P., Arnau, J., Muñoz, I., Comaposada, J., NIR technology for online determination of superficial $\mathrm{a}(\mathrm{w})$ and moisture content during the drying process of fermented sausages. Food Chem., 135 (3): 1750-5, 2012.

6. Cozzolino, D. and Murray, I., A Review on the Application of Infrared Technologies to Determine and Monitor Composition and Other Quality Characteristics in Raw Fish, Fish Products, and Seafood, Applied
Spectroscopy Reviews, 47:3: 207-218, 2012.

7. Cowe, I.A. and McNicol, J.W., The use of principal components in the analysis of near-infrared spectra. Applied Spectroscopy, 39(2): 257-266, 1985.

8. Evers, E., Nauta, M, Havelaar, A. and Henken, A., Quantitative risk assessment of food borne pathogens -a modeling approach. Chapter 4 in "New approaches to food-safety economics". Proceedings of the Frontis workshop on new approaches to food-safety economics, 35-45, 2002.

9. Hart, J., Smith, G., Verocytotoxinproducing Escherichia coli O157 outbreak in Wrexham, North Wales, July, Euro Surveill, 14 (32): 19300, 2009.

10.Horvat, K., Seregely, Zs., Andrassy, E., Dalmadi, I. and Farkas, J., A preliminary study using near infrared spectroscopy to evaluate freshness and detect spoilage in sliced pork meat. Acta Alimentaria, 37: 93102, 2008.

11.Huang, H., Liu, L., Ngadi, M.O., Prediction of pork fat attributes using NIR Images of frozen and thawed pork. Meat Sci., 119: 5161, 2016.

12.Kinoshita, K. et al., Spectral pattern of urinary water as a biomarker of estrus in the giant panda. Scientific reports, 2: 856, 2012.

13.Kitamoto, M., Kito, K., Niimi, Y., Shoda, S., Takamura, A., Hiramatsu, T., Akashi, T., Yokoi, Y., Hirano, H., Hosokawa, M., Yamamoto, A., Agata, N., Hamajima, N., Food poisoning by Staphylococcus aureus at a university festival. Jpn J Infect Dis., 62 (3): 242-243, 2009.

14.Kovacs, Z., Bázár, G., Oshimad, M., Shigeokad, S., Tanaka, M., Furukawa, A., Nagai, A., Osawa, M., Itakura, Y., Tsenkova, R., Water spectral pattern as holistic marker for water quality monitoring,Talanta, V. 147: 598-608, 2016.

15.Kumar, N., Bansal, A., Sarma, G.S., Rawal, R.K., Chemometrics tools used in analytical chemistry: An overview, Talanta, 123, 186-199, 2014.

16.Levine, P., Rose, B., Green, S., Ransom, G., Hill, W., Pathogen testing of ready-toeat meat and poultry products collected at federally inspected establishments in the United States, 1990-1999. J. Food Prot., 64:1188 -1193, 2001.

17.Li, X., Feng, F., Gao, R., Wang, L., Qian, Y., Li, C., Zhou, G., Application of near infrared reflectance (NIR) spectroscopy to identify potential PSE meat. J Sci Food Agric, 96 (9): 3148-56, 2016. 
18.Lucas, A., Andueza, D., Rock, E., Martin, B., Prediction of dry matter, fat, $\mathrm{pH}$, vitamins, minerals, carotenoids, total antioxidant capacity, and color in fresh and freeze-dried cheeses by visible-near-infrared reflectance spectroscopy. J. Agric Food Chem., 56 (16): 6801-8, 2008.

19.Lu, W., Da-Wen, S., Hongbin, P., Jun-Hu, C., Quality Analysis and Classification and Authentication of Liquid Foods by Nearinfrared Spectroscopy: A Review of Recent Research Developments, Critical Reviews in Food Science and Nutrition, DOI:10.1080/10408398.2015.1115954, 2016.

20.Matija, L., Tsenkova, R., Aquaphotomics of hydrogenated fullerenes, in: Book of Abstracts of the Second Scientific International Conference on Water and Nanomedicine, Banja Luka, 30-31, 2011.

21. Majolini, D., Trocino, A., Xiccato, G., Santulli, A., Near infrared reflectance spectroscopy (NIRS) characterization of European sea bass (Dicentrarchus labrax) from different rearing systems. Ital.J.Anim.Sci., 8 (2): 860-862, 2009.

22. Munćan, J., A comparative study of structure and properties of water by IR and Optomagnetic spectroscopy, in: Book of Abstracts of the Second Scientific International Conference on Water and Nanomedicine, Banja Luka, 56-57, 2011.

23. Naes, T. et al., A user friendly guide to multivariate calibration and classification, Chichester, UK: NIR Publications, 2002.

24.Niu, X.Y., Shao, L.M,, Dong, F., Zhao, Z., Zhu, Y., Discrimination of donkey meat by NIR and chemometrics (Article in Chinese). Guang Pu Xue Yu Guang Pu Fen Xi, 34 (10): 2737-42, 2014.

25.Prieto, N., Roehe, R., Lavín, P., Batten, G., Andrés, S., Application of near infrared reflectance spectroscopy to predict meat and meat products quality: A review. Meat Sci., 83 (2): 175-186, 2009.

26. $\mathrm{R}$ Core Team, R: A Language and Environment for Statistical Computing, 2014.

27.Reis, M.M., Rosenvold, K., Early on-line classification of beef carcasses based on ultimate $\mathrm{pH}$ by near infrared spectroscopy. Meat Sci., 96 (2 Pt A): 862-9, 2014.

28. Sandorfy, C., Buchet, R., Lachenal, G., Principles in molecular vibrations for NearInfrared spectroscopy. In Near Infrared spectroscopy in food science and technology, John Wiley \& Sons, Inc. Canada, 11-48, 2007.

29.Schmid, D., Gschiel, E., Mann, M., Huhulescu, S., Ruppitsch, W., Bohm, G., Pichler, J., Lederer, I., Hoger, G., Heuberger, S., Allerberger, F., Outbreak of acute gastroenteritis in an Austrian boarding school, Euro Surveill., 12 (3): 224, 2007.

30. Slavchev, A., Kovacs, Z., Koshiba, H., Nagai, A., Bázár, G., Krastanov, A., Kubota, Y., Tsenkova, R., Monitoring of Water Spectral Pattern Reveals Differences in Probiotics Growth When Used for Rapid Bacteria Selection. PLoS One., 10 (7), 0130698, 2015.

31. Sun, D. (ed.), Infrared Spectroscopy for Food Quality Analysis and Control. Academic Press, Burlington, MA, USA, 2009.

32. Teixeira dos Santos, C.A., Lopo, M., Pascoa, R., Lopes, J.A., A Review on the Applications of Portable Near-Infrared Spectrometers in the Agro-Food Industry, Applied Spectroscopy, 67: 1215-1233, 2013.

33. Trocino, A., Xiccato, G., Majolini, D., Tazzoli, M., Bertotto, D., Pascoli, F., Palazzi, R., Assessing the quality of organic and conventionally-farmed European sea bass (Dicentrarchus labrax). Food Chemistry, 133: 427-433, 2012.

34. Tsenkova, R., Method for Analysis with Visible and Near-infrared Spectroscopy. Patent Application No. 2004-065975, 2004a.

35. Tsenkova, R., Iordanova, I., Toyoda, K., Brown, D., Prion protein fate governed by metal binding, Biochemical and Biophysical Research Communications, 325: 1005-1012, 2004b.

36. Tsenkova, R., Aquaphotomics: Exploring Water-Light Interactions for a Better Understanding of the Biological World (part 1), NIR news, 17(3): 11-12, 2006.

37. Tsenkova, R., Introduction to Aquaphotomics, dynamic spectroscopy of aqueous and biological systems describes peculiarities of water, Journal of Near Infrared Spectroscopy, 17: 303-314, 2009.

38. Tsenkova, R., Aquaphotomics: Water in the biological and aqueous world scrutinised with invisible light. Spectroscopy Europe, 22(6): 6-10, 2010.

39. Tukey, J., Exploratory data analysis, Reading, MA: Addison-Wesley, 1977.

40. Weeranantanaphan, J., Downey, G., Allen, P. and Da-Wen, S., A review of near infrared spectroscopy on muscle food analysis: 20052010. J. Near Infrared Spectros., 19: 61-104, 2011.

41.Wu, X., Song, X., Qiu, Z., He, Y., Mapping of TBARS distribution in frozen-thawed pork using NIR hyperspectral imaging. Meat Sci., 113: 92-6, 2016.

42.Xiong, Z., Sun, D.W., Pu, H., Xie, A., Han, Z., Luo, M., Non-destructive prediction of thiobarbituricacid reactive substances (TBARS) value for freshness evaluation of chicken meat using hyperspectral imaging. Food Chem. 179: 175-81, 2015. 